\title{
An Approach for Power Optimization of Tethered UAV
}

\author{
Vimal Raj $\mathrm{V}^{1}$, Nidhi Raj $\mathrm{R}^{2}$, Jagan Kumar $\mathrm{K}^{3}$ \\ ${ }^{1}$ (Avionics Engineer/CEO, Garuda Aerospace Pvt. Ltd, India) \\ ${ }^{2}$ (Head-Avionics, Garuda Aerospace Pvt. Ltd, India) \\ ${ }_{3}^{3}$ (Electronics Engineer, Garuda Aerospace Pvt. Ltd, India)
}

\begin{abstract}
This research paper addresses the power optimization problem of a tethered quadcopter for mini payload long time hovering is expected. Tethered quadcopters are unmanned aerial vehicles with ability of vertical takeoffs, landings and hovering at a desired location. Since the vehicle is tethered, it has no flight time restrictions that the battery operated unmanned aerial vehicle has, and so suitable for long time uninterrupted applications. In already existing tethered UAV's the power consumption is not optimum due to the lack of proper knowledge about the power parameters like voltage, current and throttle level. So we concentrated on finding the optimum value of parameters to be fed to the quadcopter for a particular payload value.
\end{abstract}

Keywords: Tethered flight, UAV, Power optimization, Quadcopter

\section{Introduction}

Unmanned Aerial Vehicles are used in many research applications in scientific community. The use of battery powered unmanned aerial vehicles are quite common among hobby flyers and aerial photographers. The main problem with battery operated aerial vehicle is its flight time. A tethered UAV is capable of solving this issue. The word tethered means tied to a rope or more making restricting the movements. A tethered UAV consists of all the basic components a normal UAV needs except an onboard power source. Tethered UAV serves as an in-the -air platform for many applications while the power required for motors to generate lift is supplied by the external power source. Though the movements of such a UAV are restricted, it can be applied on long endurance required applications such as Surveillance, communication etc. The power supply for such a system is given by a UPS assembly which is capable of varying its output voltage and current depending upon load.

Garuda Aerospace has designed and developed a tethered UAV, a quadcopter with the specifications detailed in this paper.

\section{Hardware and Powering}

A quadcopter was designed and built using composite materials and aluminium rods. Designing and fabrication stages were carefully planned so as to reduce weight of frame which is the primary method to reduce power consumption. Once the basic stuructre is ready, all avionic components which comprisees of Flight controller, GPS module, Motors and ESC's are mounted. All power and communication links were established and verified. A 5 Meter long cable is used to power the UAV from UPS supply. The UPS module is selected according to the voltage requirements of the system. This supply system should be capable of varying voltage level with necessary current output. Inorder to measure the power consumption a watt meter, with matching configuration has been used.

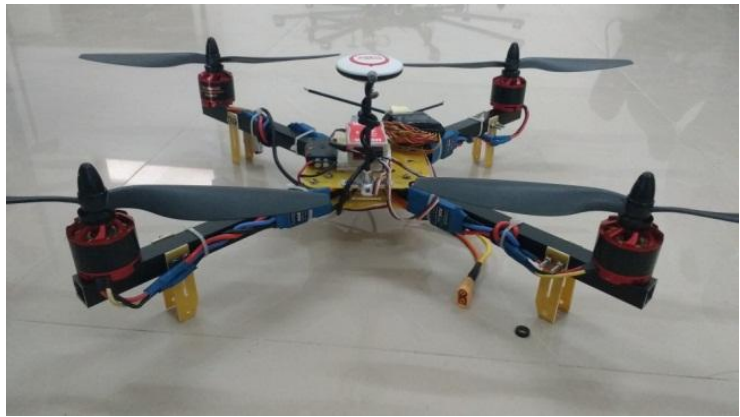

Figure 1: Quadcopter ready for testing

UPS supplies with following specifications were utilized for the purpose of powering.

Input: $220 \mathrm{~V} / 50 \mathrm{~Hz} \sim$

Output: 13.8 V- 18V DC / 30 A, Variable output 


\section{Testing}

The assembled quadcopter was tested in an indoor lab facility after ensuring the safety. Power supply is connected through UPS and input voltage is fixed at $16 \mathrm{~V}$ for testing purpose. Response of all control sticks was verified before starting the whole process. A watt meter is used to measure the consumed power during each stage of flight.

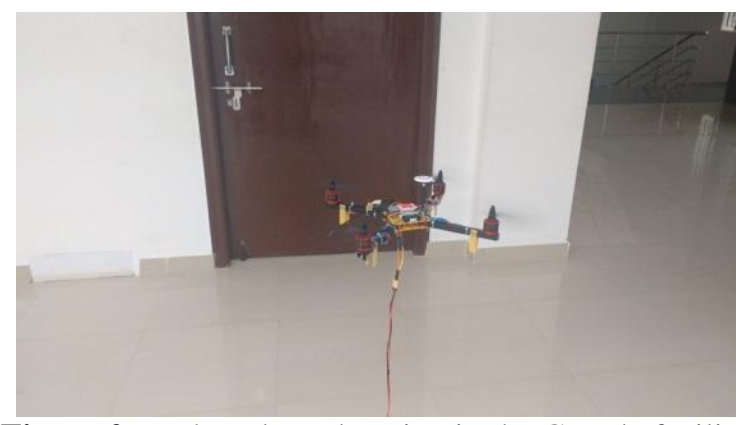

Figure 2: Tethered quad testing in the Garuda facility

\section{Observation}

The power required for just lift off from the ground is termed as Lift-off power here. Lift off power is considered as a sample of power values for each flight. For the purpose of comparison of flights at each voltage, lift off power is taken as variable.The input power is noted down for some particular throttle positions. TABLE 1 shows an observation for input voltage of $15.60 \mathrm{~V}$.

Table 1: Power consumed for each value of input, Input voltage $=15.60$

\begin{tabular}{|l|l|l|l|l|}
\hline Throttle position & Condition of UAV & Current(A) & Voltage(V) & Power(W) \\
\hline Open Circuit & Grounded & 0.3 & 15.60 & 4.6 \\
\hline Load Connected & Grounded & 0.6 & 15.54 & 9.3 \\
\hline Armed \& 10\% & Grounded & 5.2 & 15.41 & 80.01 \\
\hline $40 \%$ & Grounded & 5.9 & 15.35 & 89.2 \\
\hline $45 \%-50 \%$ & Lifting & 7.2 & 15.28 & 110 \\
\hline $60 \%$ & Hovering & 9.0 & 15.25 & 136.4 \\
\hline $60 \%$ above & No Change \\
\hline
\end{tabular}

The power consumption varies according to the stages of flight. Clearly the observation shows that after the throttle reaching $60 \%$ there is no change in altitude or power consumed. This is because of the insufficient input voltage. Further the voltage is varied up to $16.3 \mathrm{~V}$ stage by stage and similar observation is repeated.

Following graph shows the liftoff power corresponding to each input voltage. The input voltage is varied from 15.6 to 16.3. For each of this input voltage throttle is varied from 0 to $60 \%$ corresponding power is noted down. Following graph shows the lift off power for each value of input.

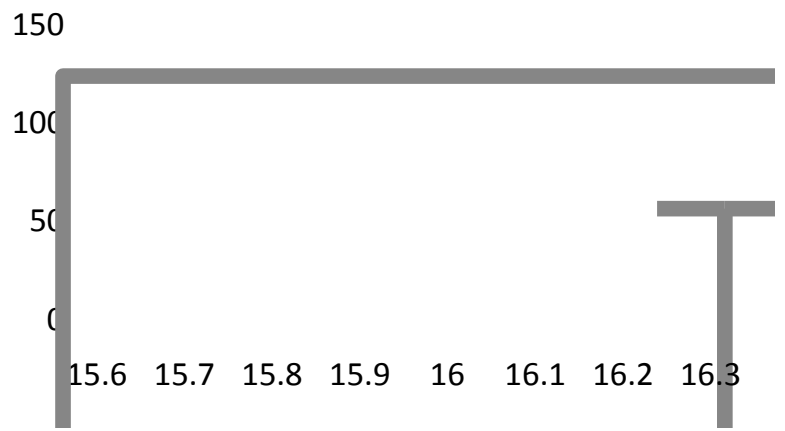

Graph 1: Input voltage v/s Lift-off power

From the observation it is clear that minimum power is consumed when input voltage is $15.7 \mathrm{~V}$. When lift off power is low, the consumed power during the whole flight is also minimized. That is also noticeable from the observed data. And the minimum power corresponding to $15.7 \mathrm{~V}$ is $86.2 \mathrm{~W}$ at $35 \%$ throttle. 


\section{Conclusion}

The tethered quad when designed for $3 \mathrm{~S}$ battery, consumes less power when the input voltage reaches 15.7 Volts and the corresponding lift-off power is 86 Watts. Take-off happens at $35 \%$ throttle irrespective of the craft weight and dimensions. This value is the optimum position for throttle. A further investigation is needed for giving out the explanation for optimum power at each stage of flight. The power consumed by a model depends on its weight and aerodynamics. The power can be further reduced by using much lighter materials and efficient motors.

\section{Acknowledgements}

The authors would like to acknowledge the support of Garuda Aerospace research engineers for the throughout help support. And the Almighty for his grace.

\section{References}

[1]. Tethering System for Unmanned Aerial Vehicles, Quentin Morales-Perryman (Hampton University, Electrical Engineering), SUNFEST Fellow, Dr. Daniel D. Lee, Department of Electrical Systems Engineering

[2]. Unmanned Aerial Vehicles for Power Line Inspection: A Cooperative Way in Platforms and Communications, Chuang Deng , Shengwei Wang, Zhi Huang, Zhongfu Tan and Junyong Liu, Journal of Communications Vol. 9, No. 9, September 2014

[3]. Uninterruptible Power Supply Design for Heavy Payload Tethered Hexaroters, Guangwei Wang, , Wasantha Samarathunga, , Shiqin Wang, International Journal of Emerging Engineering Research and Technology Volume 4, Issue 2, February 2016, PP 1621 ISSN 2349-4395 (Print) \& ISSN 2349-4409 (Online) 